\title{
Geostatistical Analysis of the Spatiotemporal Dynamics of Powdery Mildew and Leaf Rust in Wheat
}

\author{
Jonas Franke, Steffen Gebhardt, Gunter Menz, and Hans-Peter Helfrich
}

First author: Center for Remote Sensing of Land Surfaces, Walter-Flex-Strasse 3, University of Bonn, 53113 Bonn, Germany; second author: Department of Crop Science and Resource Conservation INRES-Crop Science and Plant Breeding, Katzenburgweg 5; third author: Department of Geography, Remote Sensing Research Group, Meckenheimer Allee 166; and fourth author: Mathematical Seminar, Nussallee 15, University of Bonn, 53115 Bonn, Germany.

Accepted for publication 27 March 2009.

\begin{abstract}
Franke, J., Gebhardt, S., Menz, G., and Helfrich, H.-P. 2009. Geostatistical analysis of the spatiotemporal dynamics of powdery mildew and leaf rust in wheat. Phytopathology 99:974-984.

Plant diseases are dynamic systems that progress or regress in spatial and temporal dimensions. Site-specific or temporally optimized disease control requires profound knowledge about the development of each stressor. The spatiotemporal dynamics of leaf rust (Puccinia recondite $\mathrm{f}$. sp. tritici) and powdery mildew (Blumeria graminis f. sp. tritici) in wheat was analyzed in order to evaluate typical species-dependent characteristics of disease spread. During two growing seasons, severity data and other relevant plant growth parameters were collected in wheat fields. Spatial characteristics of both diseases were assessed by cluster analyses

into four classes of temporal dynamics. The results of the spatial analysis showed that both diseases generally occurred in patches but a differentiation between the diseases by their spatial patterns and spread was not possible. In contrast, temporal characteristics allowed for a differentiation of the diseases, due to the fact that a typical trend was found for leaf rust which differed from the trend of powdery mildew. Therefore, these trends suggested a high potential for temporally optimized disease control. Precise powdery mildew control would be more complicated due to the observed high variability in spatial and temporal dynamics. The general results suggest that, in spite of the high variability in spatiotemporal dynamics, disease control that is optimized in space and time is generally possible but requires consideration of disease- and case-dependent characteristics.
\end{abstract} using spatial analysis by distance indices, whereas the temporal epidemic trends were assessed using statistical parameters. Multivariate statistics were used to identify parameters suitable for characterizing disease trends
Additional keywords: epidemics, SADIE.
Powdery mildew (Blumeria graminis f. sp. tritici) and leaf rust (Puccinia recondite f. sp. tritici) are among the most frequently controlled fungal diseases in cereals in Central Europe. These fungal pathogens can cause considerable yield losses $(7,10,12$, 16), particularly in wheat, the major crop in the European Union (2). Wheat is often treated with fungicides several times per growing season. For instance, an average of 2.9 fungicide treatments was applied to wheat crops in Great Britain in 2004 (6). Similar observations were made in the Netherlands in 1995 (39).

Fungal diseases often appear in patches and can cause aggregated damage to plants $(4,23,47)$. Hence, an application of pesticides on those areas where the stressors occur and actually infect the plants may be highly effective (41). However, wind-dispersed pathogens such as $B$. graminis and $P$. recondita pose the greatest problem to site-specific fungicide applications, because spores are transported over long distances, with relatively flat dispersal gradients resulting in potentially large target areas for spore deposition $(5,11,18,42)$. In order to make effective site-specific (i.e., patch- or focus-specific) disease control possible and to reduce the number and improve timing of fungicide applications, a better knowledge of the spatial and temporal variability of each disease and its pattern is fundamental (30). Spatiotemporal dynamics of plant diseases are influenced by biotic and abiotic factors and

Corresponding author: J. Franke; E-mail address: jonasfranke @uni-bonn.de

*The $\boldsymbol{e}$-Xtra logo stands for "electronic extra" and indicates that Figures 3, 4, 5, 6, 9,10 , and 11 appear in color online.

doi:10.1094/PHYTO-99-8-0974

(c) 2009 The American Phytopathological Society reflect the interaction between host, microorganism, and environment. Depending on the pathogen species, soil characteristics, topography, plant density, host resistance, host growth stage, amount of existing inoculum, temperature, humidity, and so on affect the spatial spread of plant diseases $(17,20,33,38)$.

Savary and Cooke (34) and Madden (13) stated that plant disease epidemiology leads to specific disease control recommendations and conceptual innovations in the management of plant diseases. For a more effective disease control, the optimal timing of management actions is important. Therefore, several studies focused on the first occurrence and the temporal development of plant diseases and demonstrated the potential of temporally optimized disease management strategies $(24,30,36)$. Even though excellent studies of the spatiotemporal dynamics of plant diseases in general have been conducted $(8,11,18,45,47,48)$, as well as studies of the ecology of specific foliar diseases and their requirements for environmental conditions $(17,35)$, there is a lack of epidemiological studies concerning the empirical description and quantification of spatiotemporal dynamics of the specific foliar diseases powdery mildew and leaf rust of wheat. The identification of similarities and differences between specific diseases is important for determining the course of epidemics and can help to predict yield losses $(31,45)$. Comparative studies of the temporal dimension of plant diseases are a common practice in epidemiology $(9,10)$. Many studies focus on the change in intensity over time but do not explicitly consider the spatial component (15). The aim of this study was to address key elements of powdery mildew and leaf rust epidemics, conforming to Madden's description of the purpose of epidemiology: "Epidemiology involves the study of the temporal, spatial and spatio-temporal dynamics of 
disease in populations, and the utilization of results of experiments and surveys to describe, understand, compare, and predict epidemics" (13).

Therefore, the present study focused on the temporal, spatial, and spatiotemporal dynamics of powdery mildew and leaf rust in wheat in order to (i) identify the spatial characteristics of these diseases, (ii) assess and compare their temporal dynamics, (iii) find statistical measures that are suitable to describe the temporal dynamics of observed disease progress curves, (iv) define interactions of the diseases, and (v) highlight the potential of sitespecific or temporally optimized disease control.

\section{MATERIALS AND METHODS}

Observation sites. The test sites observed in 2005 and 2006 were located at $50^{\circ} 37^{\prime} \mathrm{N}, 6^{\circ} 59^{\prime} \mathrm{E}$ at an altitude of $175 \mathrm{~m}$ above sea level. The annual mean temperature is $9.2^{\circ} \mathrm{C}$ with $600 \mathrm{~mm}$ of rainfall and 1,534 sun hours per year. The field plots (a 5.7-ha field in 2005 and a 4.6-ha field in 2006) were chosen based on their flat topography.

In 2005, the observed field plot with winter wheat was divided into three subareas that received different fungicide applications in order to ensure a range in disease severity. To ensure that at least one area remained disease free, plot 1 (1.2 ha) was treated with fungicides twice, on 11 May 2005 at $\mathrm{BBCH}$ decimal growth stage (GS) 34 (46) and on 9 June 2005 at GS 57. A mixture of the fungicides Opus Top (BASF, Ludwigshafen, Germany), with the active ingredients epoxiconazole and fenpropimorph at a dose rate of 0.8 liter ha ${ }^{-1}$, and Flexity (BASF), with the active ingredient metrafenone at a dose rate of 0.4 liter $\mathrm{ha}^{-1}$, was applied on the first application date. For the second treatment for plot 1, the fungicide Juwel Top (BASF), with the active ingredients epoxiconazole, fenpropimorph, and kresoxim, was used at a dose rate of 1.0 liter $\mathrm{ha}^{-1}$. To cause a reduced or delayed infection in plot 2 (0.8 ha), it was only treated once (on 11 May 2005 at GS 34). The focus of this study was fungicide-untreated plot 3 , which was the largest (3.7 ha), so that the fungal infections could spread naturally. In 2006, the entire observed field was not treated with fungicides, in order to have similar conditions to that of plot 3 of the previous year. Without artificial inoculation, powdery mildew and leaf rust appeared in the stands. To reduce the presence of undesirable stress factors such as nitrogen deficiency or weed infestation, all plots were treated uniformly with fertilizers and herbicides.

In 2005, in-field data were regularly collected at 50 sample points (their proportional distribution was predefined in a Geographic Information System) whose exact positions were defined by differential GPS readings taken in field. At 10 dates from 21 April 2005 (GS 30) until 23 June 2005 (GS 73), severity (percentage of diseased leaf area) of each disease was estimated; GS and growth height was measured. Severity was estimated on a whole-plant basis within an area of $1 \mathrm{~m}^{2}$ using a predefined rating scale scheme that gives percentages of diseased leaf area. Even though disease assessment has received considerable attention $(15,22)$, there is no standardized method to measure disease severity, and visual estimations are subject to uncertainty (25), which is a component of any observed variance in disease assessments.

Digital images (nadir taken with a Canon PowerShot A95) were acquired at each sample point, whereby an area of $\approx 1 \mathrm{~m}^{2}$ was covered by the images. Vegetation pixels in the images were identified by a supervised image classification procedure and the percentage of vegetation cover was determined. Soil characteristics were investigated by EM38 measurements of the apparent electrical conductivity (ECa) (Geonics Limited, Ontario, Canada), which is a useful tool to obtain information about soil heterogeneity. In all, $\approx 92 \%$ of the measured values were 20 to $30 \mathrm{mS} \mathrm{m}^{-1}$, indicating a very homogeneous soil texture of loamy silt (21).
Hence, due to the minimization of other stress variables by adequate treatments and the indicated homogeneous soil conditions, it could be assumed that the spread of the diseases was only marginally affected by other stress factors. In 2006, similar observations were done at 54 sample points in a winter wheat field. Due to the fact that, in this growing season, the epidemics progressed much more slowly than in 2005, in-field data was collected at four dates only (from 20 April 2006 [GS 29] until 14 June 2006 [GS 61]).

Analysis of the spatial dynamics of powdery mildew and leaf rust. In order to analyze the spatial pattern of the diseases, the spatial analysis by distance indices (SADIE) methodology introduced by Perry (26) was applied to the observed severity data. SADIE is now being used more frequently in plant pathology for analyzing disease patterns (44). Detailed descriptions of SADIE have been presented in previous studies (15,26-29). Additionally, SADIE has been applied successfully in the past to analyze spatial distributions of field pests such as insects, diseases, and weeds $(1,30,31,36,37,40,43)$. SADIE can be seen as an alternative approach to the autocorrelation- and semivariancebased methods that are also used to analyze spatial count data. The results of SADIE are often in good agreement with the results of these methods but also reveal differences $(15,44)$.

SADIE provides an overall measure of spatial aggregation of count data values, $c$, in data sets with spatially referenced space. Values of $I_{a}>1$ indicate aggregated pattern. In addition to this aggregation measurement of a complete data set, SADIE aims for the more detailed identification and measurement of clusters in spatial data and provides a dimensionless cluster index, $v$, for each sample point, giving the degree of affiliation to a cluster. It is distinguished between two types of clusters: (i) patches $\left(v_{i}\right)$ which constitute neighborhoods of relatively high values that are larger than the sample mean, $m$, and (ii) gaps $\left(v_{j}\right)$ that are distinguished by neighborhoods of relatively low values that are $<m$. For random arrangements of the values $c$, the expectation of $v_{i}$ is 1 and a sample point that is part of a patch is indicated by $v_{i}>1$, whereas the expectation of $v_{j}$ is -1 and a sample point belonging to a gap has an index value $v_{j}<-1(26,27)$. In epidemiology, a cluster is generally defined as a number of diseased plants grouped together (19).

The results of SADIE were cluster index values for each sample location, each disease, and date, which were subsequently interpolated applying ordinary kriging (spherical model). For the interpolation, the software ArcGIS 9.0 (ESRI, Redlands, CA) was used and the cluster indices were contoured (by isolines of samecluster index values). These contour maps generally allow for an easy recognition of patches and gaps (15).

Analysis of the temporal dynamics of powdery mildew and leaf rust. An analysis of the temporal dynamics of epidemics gives information about the occurrence of diseases, their progress, and possible trends $(9-11,14,47)$. Analyses of disease progress curves (DPCs) are powerful tools to represent the temporal, spatial, and spatiotemporal dynamics of diseases (13). By comparing DPCs, it is often possible to classify different shape types of the curves via growth functions or variables describing epidemics $(10,14)$. For each type of DPC, different variables as described by Kranz (10) and Madden and Campbell (14) are suitable to classify epidemics. Mathematical expressions were developed that are either useful for describing entire DPCs or some curve elements. Kranz (9) also stated that any disease can have various DPCs, depending on the influencing factors. The focus of the present study was to define statistical parameters which might be useful to describe the dynamics of any curve representing the progress of any phenomenon. This rather general approach allows for a description of entire DPCs, independent of the disease or shape type. For the 2005 data, the following parameters were calculated for each powdery mildew and leaf rust progress curve at each sample point $(n=50)$ (powdery mildew and leaf rust separately): 
(i) Mean

(ii) Standard deviation

(iii) Coefficient of variation

(iv) Root-mean-square variation; $d_{i-1}$ is the number of days between observed severity $x_{i}$ and $x_{i-1}$

$$
\omega_{x}=\sqrt{\frac{1}{n} \sum_{i=2}^{n}\left(\frac{x_{i}-x_{i-1}}{d_{i-1}}\right)^{2}}
$$

(v) Relative number of curve intersections of the mean; where $c_{m i}$ is the number of curve intersections of the mean

$$
c_{r}=\frac{c_{m i}}{n-1}
$$

(vi) Total variation

$$
\Delta_{t}=\sum_{i=2}^{n}\left|x_{i}-x_{i-1}\right|
$$

(vii) Mean total variation

$$
\overline{\Delta_{t}}=\frac{1}{n} \sum_{i=2}^{n}\left|x_{i}-x_{i-1}\right|
$$

(viii) Mean of absolute deviations from the linear regression $f(x)=$ $a x+b$

$$
\bar{D}=\frac{1}{n} \sum_{i=1}^{n}\left|x_{i}-f\left(x_{i}\right)\right|
$$

A classification of the different temporal dynamics of the diseases was applied to the data collected in 2005 by visual assessments. The 100 observed DPCs (50 for each disease) were categorized into four predefined classes describing the main temporal dynamics based on their shapes. The first class, 'stable', characterized DPCs with temporally constant severity values. The class 'fluctuating 1' represented more dynamic DPCs with lower severity and the class 'fluctuating 2' represented curves showing the same characteristics with higher severities. DPCs that show strongly increasing trends were assigned to class 'increasing'. Afterward, a feature selection procedure was applied to the statistical data of all DPCs using the commonly available decision tree software see5 (RuleQuest Research Pty. Ltd., NSW, Australia), in order to select those of the calculated statistical parameters that are really relevant to classify DPCs into these four classes. The decision tree learning algorithm is a commercial decision tree and rule induction engine developed by Quinlan (32). The input for this procedure was a training data set containing the eight above- mentioned statistical parameters for each of the 100 manually categorized DPCs. The output indicated relevant parameters that help to describe the temporal dynamics of epidemics.

Analysis of the spatiotemporal dynamics of powdery mildew and leaf rust. For the spatiotemporal analysis of the disease spread, the spatial development of disease patches detected by SADIE during the growing periods was used. In order to investigate the spatial dynamics of the diseases, only the identified patches were further analyzed, because gaps are characterized by low values (i.e., healthy plant areas). By applying a threshold to the interpolated cluster indices $(=1)$, patches were selected that corresponded to areas showing aggregated high severities. In order to analyze their spatiotemporal dynamics, some patch parameters such as the total patch size and the maximal cluster index were calculated and the temporal patterns of these statistics were monitored.

In order to assess possible interrelationships between powdery mildew and leaf rust epidemics in 2005, the overall spatial association $X$ (mean of the local spatial association values at each sample point) of the diseases was analyzed by an extension of the SADIE technique. The similarity between two patterns from the same occasion was thereby quantified by the degree of spatial association between the data sets as described by Winder et al. (43). Perry and Dixon (28) and Winder et al. (43) give a detailed description of this SADIE extension. In addition, this approach was used to quantify the spatiotemporal association of each epidemic by analyzing the spatial association of an epidemic between consecutive observation dates (i.e., as a measurement of temporal dynamics or stability of disease patches).

\section{RESULTS}

The height of the wheat canopy showed a monotonous increase in both years (Figs. 1A and 2A), whereas the percentage of vegetation cover, as derived from nadir taken images, showed a more fluctuating trend, particularly in 2005 (Fig. 1B). At GS 31, when tillering was already completed, the wheat started stem extension, which caused a lower vegetation cover fraction. Vegetation cover increased by the development of more wheat leaves from GS 34 . At later GSs (GS 41), this trend was reversed, due to an increase in wheat leaf rust severity in plot 3 as well as maturation. Particularly in the fungicide-untreated plot 3 , the strong leaf rust infection already affected the canopy structure, resulting in significantly decreased vegetation cover fractions (Fig. 1B). In 2006, this was not the case due to mostly very low disease severities (Fig. 2B).
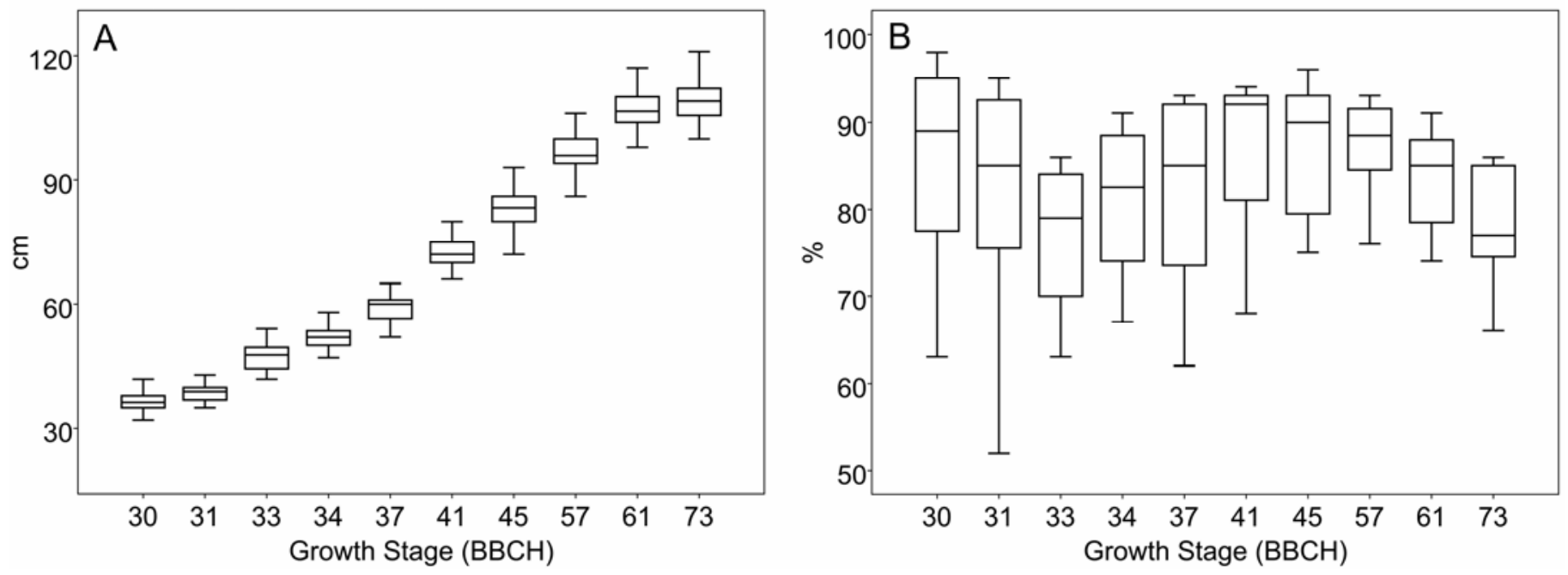

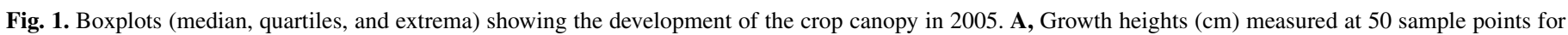
each sampling date and $\mathbf{B}$, percent vegetation cover as derived by classification of the images taken at the same locations. 
Spatial dynamics of powdery mildew and leaf rust. The SADIE results showed that both diseases occurred in patches. SADIE results for leaf rust for 2005 are displayed in Figure 3. The contours represent the cluster indices (patches $>1$ and gaps $<-1)$ as detected by the SADIE algorithm; graduated points symbolize the in-field observed severity and the gray-scale image gives the mean cluster index of all dates. Due to the fact that, in 2005, leaf rust first appeared at GS 34, the cluster index could not be calculated for earlier dates. Due to very low severities at only a few locations in the field, no leaf rust patches were identified before GS 45. With GS 45, the cluster index scored values $>1$ that are defined to be part of a patch. Two patches were identified at GS 57 and one clear patch was found for GS 61 and 73, with extreme severities up to $70 \%$. The mean cluster index of all dates indicates areas where aggregated severities were the highest. Low mean cluster index values were found in fungicide-treated plots 1 and 2. In addition, in some parts of the fungicide-untreated plot 3, low mean cluster index values were obvious as well, resulting from prevailing gaps.

The SADIE results for 2006 for leaf rust (Fig. 4) revealed similar characteristics. Leaf rust cluster indices $>1$ could not be found until GS 45, when only a small patch occurred in the western part of the field. Leaf rust patches developed by GS 61, whereby a large and some smaller patches existed. Strong gradients of the cluster indices were obvious on this date. In contrast to 2005 , only low severities occurred in 2006 , with maximum values of only $15 \%$.

The spatial pattern of powdery mildew in 2005 showed different characteristics (Fig. 5). Whereas no leaf rust infection occurred early in the season, as early as GS 30 and 31 large powdery mildew patches were identified by SADIE in the southern part of plot 3. At GS 33, these patches disappeared and a small patch in the northern part occurred. No patch was found at GS 34 and, at GS 37 and 41, only patches with lower cluster indices $(<1.5)$ were identified. A new powdery mildew patch emerged at GS 45 that grew through GS 61 and abated until GS 73. In contrast to the leaf rust severities, powdery mildew severities were generally lower, with a maximum of $30 \%$ infected leaf area at GS 31 and 57 . The mean powdery mildew cluster index revealed that, even in the fungicide-treated plots, disease patches occurred. Similar to the leaf rust results, an area in the eastern part of untreated plot 3 was identified, characterized by low cluster index values, where disease patches never occurred.

In 2006, a first powdery mildew patch occurred at GS 32 (Fig. 6). Only at GS 45, no cluster indices $>1$ were found. At GS 61, well-developed disease patches appeared in the middle part of the field which were close to each other. Similar to leaf rust severity, powdery mildew severity was also lower in 2006 than in 2005, with maximum severity values of only $8 \%$.

Temporal dynamics of powdery mildew and leaf rust. In 2005, severe leaf rust infections could be avoided in plots 1 and 2 by appropriate fungicide treatments (Fig. 7). In plot 2, which was only treated once, leaf rust severity was $<10 \%$. Leaf rust infections occurred late in the season and an exponential increase was observed in plot 3 from GS 41. At the last observation date, the infection rate increased rapidly, with maximum severities of $70 \%$. In contrast to leaf rust, powdery mildew occurred early in the season. As early as GS 30, 25\% infected leaf area was observed in some parts of untreated plot 3 (Figs. 7 and 8; outliers are not shown) and a temporally more dynamic progress of the powdery mildew epidemic was obvious (i.e., severities fluctuated throughout the season).

In 2006 (Fig. 8), due to very dry environmental conditions, leaf rust and powdery mildew developed much more slowly and showed very low maximal severities. Leaf rust occurred at GS 45, with maximal severities of only $3 \%$. At GS 61, leaf rust severities reached maxima of $15 \%$ infected leaf area. Similar to 2005, powdery mildew occurred earlier in the season. At GS 32 and 45, maximum severities of $3 \%$ were obvious whereas, at GS 61, maxima of only $8 \%$ occurred.

In order to evaluate relevant statistical parameters that describe the temporal dynamics of the diseases, first, a categorization of the 100 observed DPCs (50 for each disease) was applied to the data collected in 2005. For leaf rust, the temporal dynamics at only four sample points were categorized as dynamic with low severities (fluctuating 1) and at three sample points it was defined as dynamics with high severities (fluctuating 2). In all, 11 DPCs were categorized as stable, whereas 32 of 50 leaf rust progress curves showed an increasing trend. For powdery mildew, the temporal dynamics at 23 sample points were categorized as fluctuating 1 and 10 were categorized as fluctuating 2, whereas 9 of these sample points were localized in plot 3 . The disease progress at 17 sample points were categorized as stable; no increasing curves were found for powdery mildew. The feature selection procedure that was applied to the statistical parameters of the observed DPCs showed that only the following five of the eight statistical parameters were relevant to discriminate four classes of temporal disease dynamics (sorted by their importance): (i) relative number of curve intersections of the mean, (ii) total variation, (iii) mean of absolute deviations from
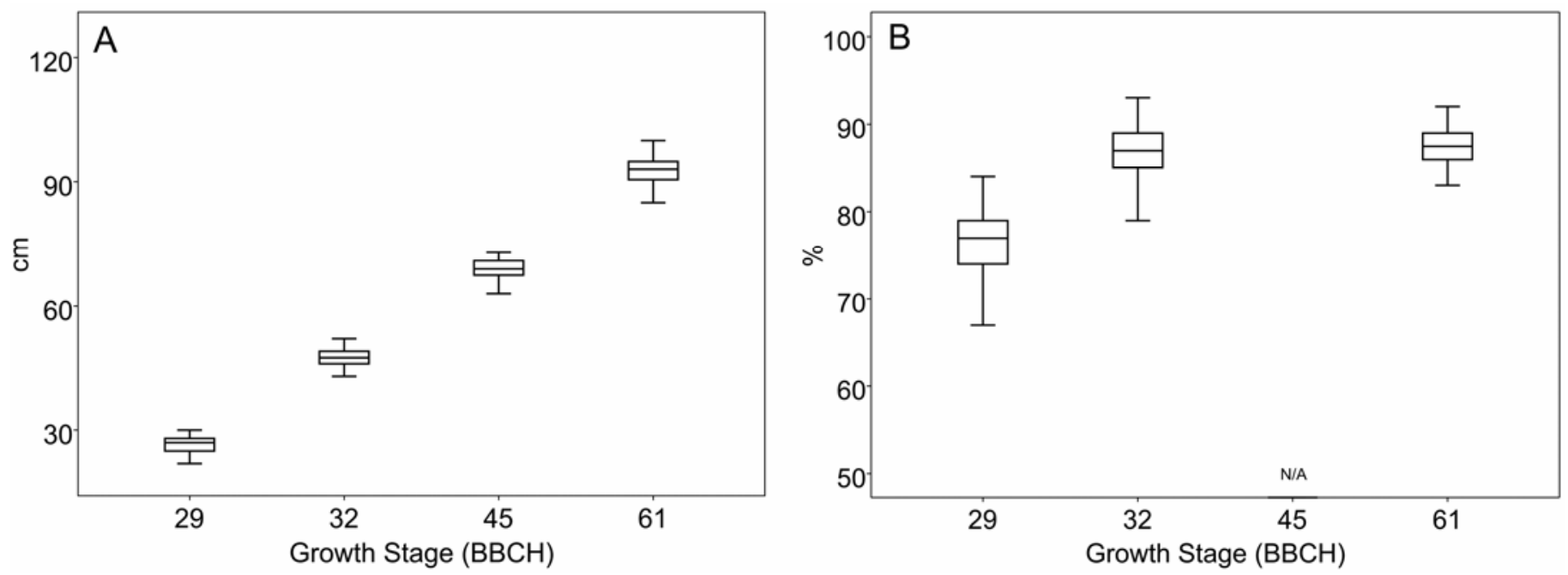

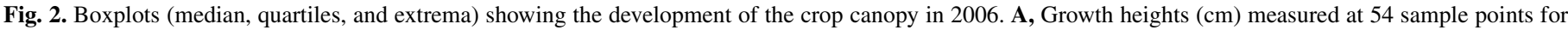

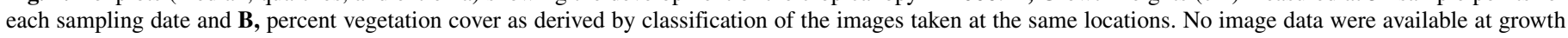
stage 45 which could be used for classification. 
the linear regression, (iv) standard deviation, and (v) coefficient of variation.

The mean, mean total variation, and root-mean-square variation could not be identified as relevant parameters for a classification of the temporal dynamics. By the use of the selected statistical parameters, a detailed description and classification of multitemporal disease severity data is possible that allows for an assessment of typical differences between powdery mildew and leaf rust progresses.

Spatiotemporal dynamics of powdery mildew and leaf rust. In 2005, the trends of disease incidence (percentage of observed infection cases found at the sample points) in fungicide-untreated plot 3 (Fig. 9) were processed similarly to the disease severity trends. At GS 30, powdery mildew infections were found at $85 \%$ of all sample points. In contrast, no leaf rust infections were found until GS 34 but, from then on, the disease spread continuously. The number of locations with powdery mildew infections varied and declined from GS 57. In 2006, locations of leaf rust infections increased exponentially, whereas powdery mildew spread slower.

Disease patches as identified by SADIE were further analyzed in order to ascertain spatiotemporal trends for each disease in 2005. Therefore, the total patch size in hectares and the maximal cluster index was determined. Courses of leaf rust and powdery mildew patch characteristics are shown in Figure 10. Similar to the temporal characteristics of a powdery mildew epidemic, the dynamic of the spatial dimension of this disease shows more variation than those of leaf rust. Powdery mildew patches occur much earlier in the season, which collapse and rebuild over the season. Nevertheless, some powdery mildew infections occurred
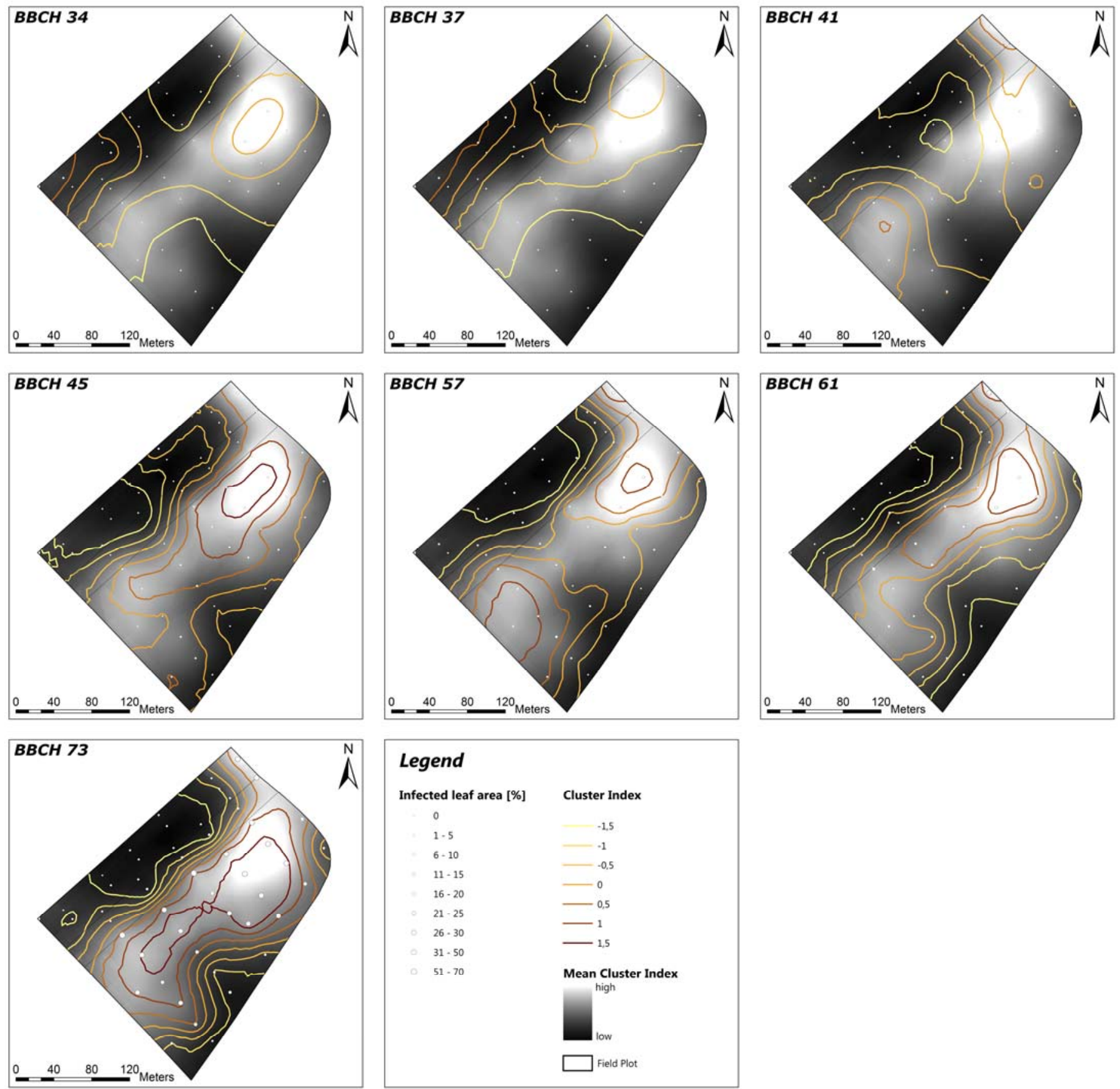

Fig. 3. Interpolated and contoured leaf rust cluster indices for each observation date in 2005 . The map represents patches and gaps as identified by spatial analysis by distance indices. Graduated points symbolize the in-field observed severities and grayscale images give the mean cluster index of all dates. 
at GS 34 but did not appear in patches. The time courses of the total patch size and maximal cluster index were similar in both cases, with two peaks for powdery mildew at GS 31 and 61 .

The test of spatial association between powdery mildew and leaf rust showed a positive association at all dates when both diseases occurred. Maximal overall association with an $X$ value of 0.51 was found at high leaf rust infection stages (GS 73).

The quantification of the overall temporal association of each epidemic, by analyzing the spatial association of an epidemic between consecutive observation dates, showed positive $X$ values at all dates for both diseases (Fig. 11). Lowest temporal associations of powdery mildew patches were obvious between GS 37 and 41 , when severity declined. Temporal stability of leaf rust was lowest at early infection stages.

\section{DISCUSSION}

In the present study, the interrelationship between spatial disease aggregation and temporal progress was analyzed for powdery mildew and leaf rust in wheat. General differences were found in the spatial as well as in the temporal dimension. In 2005, the first leaf rust patch was identified by SADIE at GS 45 . This patch was spatially constant over the growing season, which corresponded to high mean cluster indices at this location. In contrast, powdery mildew patches that occurred early in the season were more dynamic. Some powdery mildew patches disappeared and new patches grew at different locations during the observed period. The manifestation of patchiness in epidemics of diseases is generally an important fact for spatially adjusted management actions. However, powdery mildew patches were not existent at all observation dates. For instance, no patch was obvious at GS 34 in 2005, although some powdery mildew infections were observed with severities up to $10 \%$. The analysis of the inherent temporal stability of both diseases via an association test between consecutive observation dates indicated a temporal correlation in all cases (positive $X$ values). Aside from this revealed stability of diseases at certain locations, diseases nevertheless spread. Madden et al. (15) and Lucas (11), among others, have emphasized that an increase of epidemics in time generally occurs with a spread in space. However, does this conversely mean that decreasing severities correspond to a decrease in disease-affected space? Our observations for powdery mildew in 2005 suggest this was the case (Figs. 7, 9, and 10). The apparent decrease in disease severities and reduction of infected areas could have several causes. Either new leaves emerged so that powdery mildew severity (quantified at whole-plant basis) decreased because plants outgrew the disease, or temporarily unfavorable environmental conditions for powdery mildew prevailed. These cases could have resulted in the reduction in severity or even disappearance of disease patches. In general, powdery mildew occurred on an
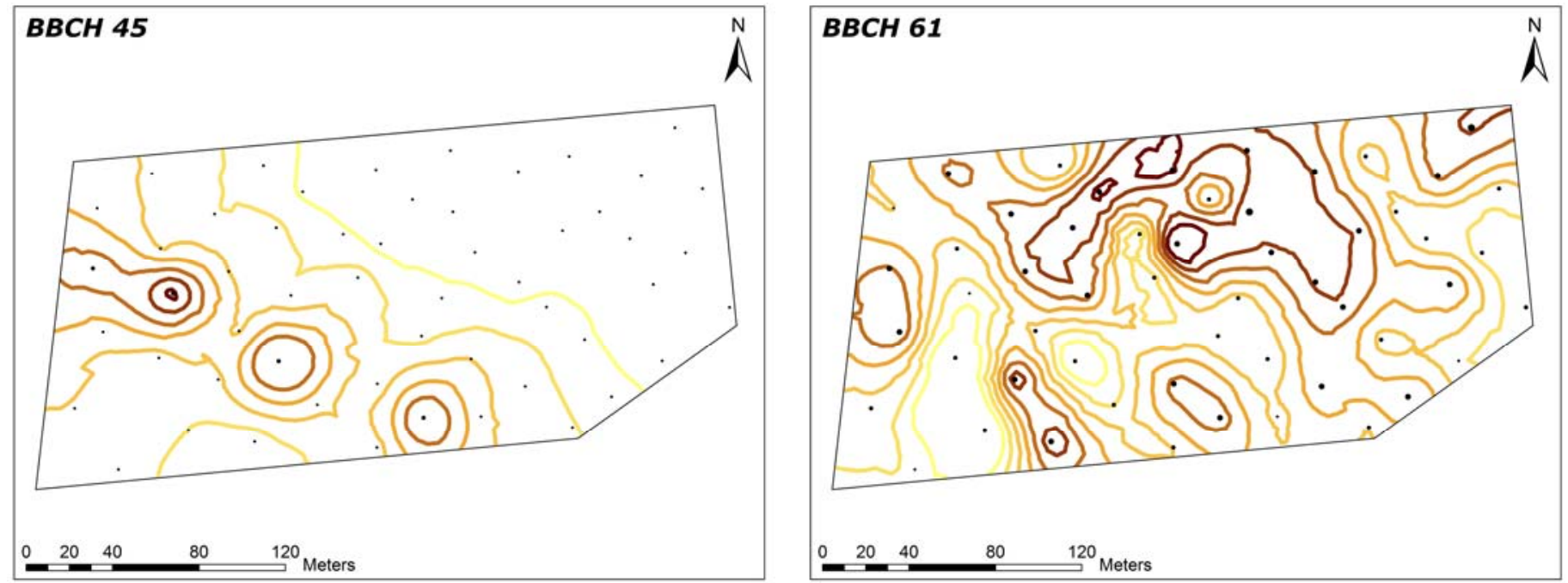

\section{Legend}

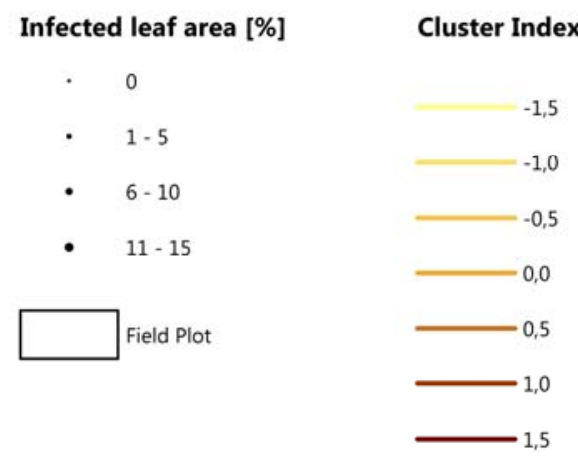

Fig. 4. Interpolated and contoured leaf rust cluster indices for each observation date in 2006. The map represents patches and gaps as identified by spatial analysis by distance indices. Graduated points symbolize the in-field observed severities. In contrast to the data of 2005 , the mean cluster index was not calculated due to a low sampling rate $(n=4)$. 

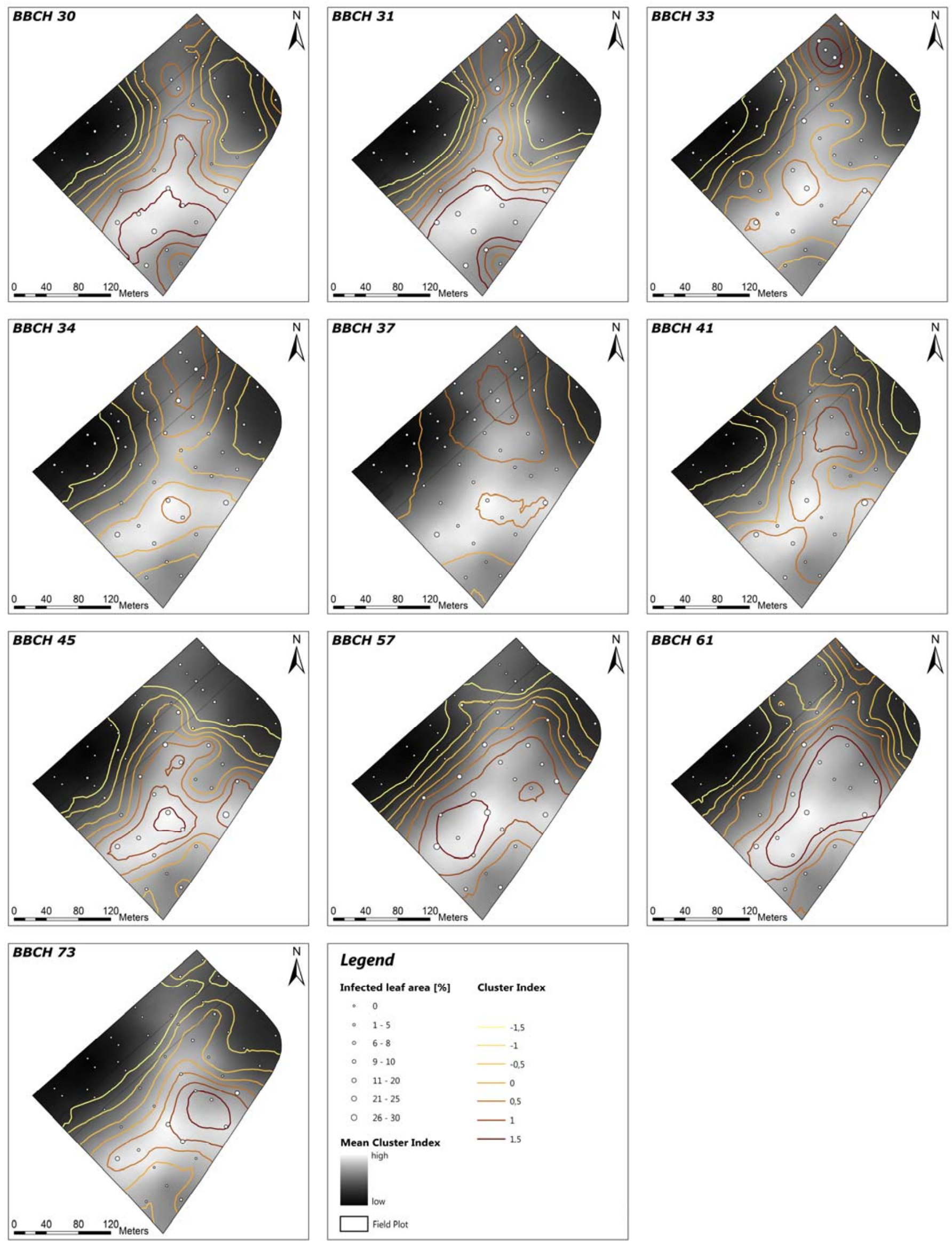

Fig. 5. Interpolated and contoured powdery mildew cluster indices for each observation date in 2005 . The map represents patches and gaps as identified by spatial analysis by distance indices. Graduated points symbolize the in-field observed severities and grayscale images give the mean cluster index of all dates. 
actively growing wheat plant whereas leaf rust developed later, at a time when vegetative growth had stopped or was slowing. The spatial dynamics of powdery mildew were strongly related to its temporal dynamics. The progress of leaf rust observed in this study conformed to the description in the literature, with an initial latent period followed by an exponential increase, whereas powdery mildew severity was found to fluctuate, particularly in 2005, when overall severities were higher than in 2006. The relatively late occurrence of leaf rust compared with powdery mildew has been noted previously (3). Even though higher maximal severities of $70 \%$ were observed for leaf rust, the overall cumulative impact of powdery mildew on the hosts might have been higher due to the longer period in which the disease could affect the plant.

Descriptive terms can be used to compare the shape of observed disease trends (10). As discussed by Madden (13), even though the utilization of mathematical and statistical methods complicates the analysis of epidemics, they are foundations for understanding epidemics. Therefore, DPCs were the basis for a statistical determination of powdery mildew and leaf rust epidemics. Statistical parameters were chosen that are suitable to analyze trend dynamics, generally applicable to any epidemic. A categorization and description of the DPCs allowed for a differentiation of the diseases via classification of their course with relevant statistical parameters that were assessed by a feature selection procedure.
In 2005, the observed DPCs in plot 3 at later crop GSs (from GS 57), particularly the opposing trends of severity (Fig. 7), percentage of infections at sample points (Fig. 9), and patch characteristics (Fig. 10), indicate possible negative correlation of diseases. Tests of the overall spatial association between powdery mildew and leaf rust indicated that such negative association occurred. However, because severity was assessed on a whole plant basis, there is no means to establish from our data whether leaf rust replaced powdery mildew on the same infected leaves or if it colonized younger leaves than powdery mildew.

Regarding potential disease control strategies, our results lead to the assumption that a spatiotemporally adjusted control of epidemics with monotonic increase in time and space, such as leaf rust, may be safer and rather efficient. In 2005, by reducing the number of fungicide treatments to one per season as applied in plot 2, severe leaf rust infections could be avoided. In contrast, site- or time-specific control of powdery mildew seems to be more complicated due to higher spatiotemporal dynamics of this disease. However, the spatial patterns of the diseases-occurrence in patches-generally allow for a site-specific adjustment of the application rate. The control of polycyclic pathogens with fungicides decreases infection severity, depresses lesion expansion, and reduces sporulation and, thus, the disease cycle is slowed down, because the latent period between infection and sporulation is increased by preventing the pathogens from generating fresh
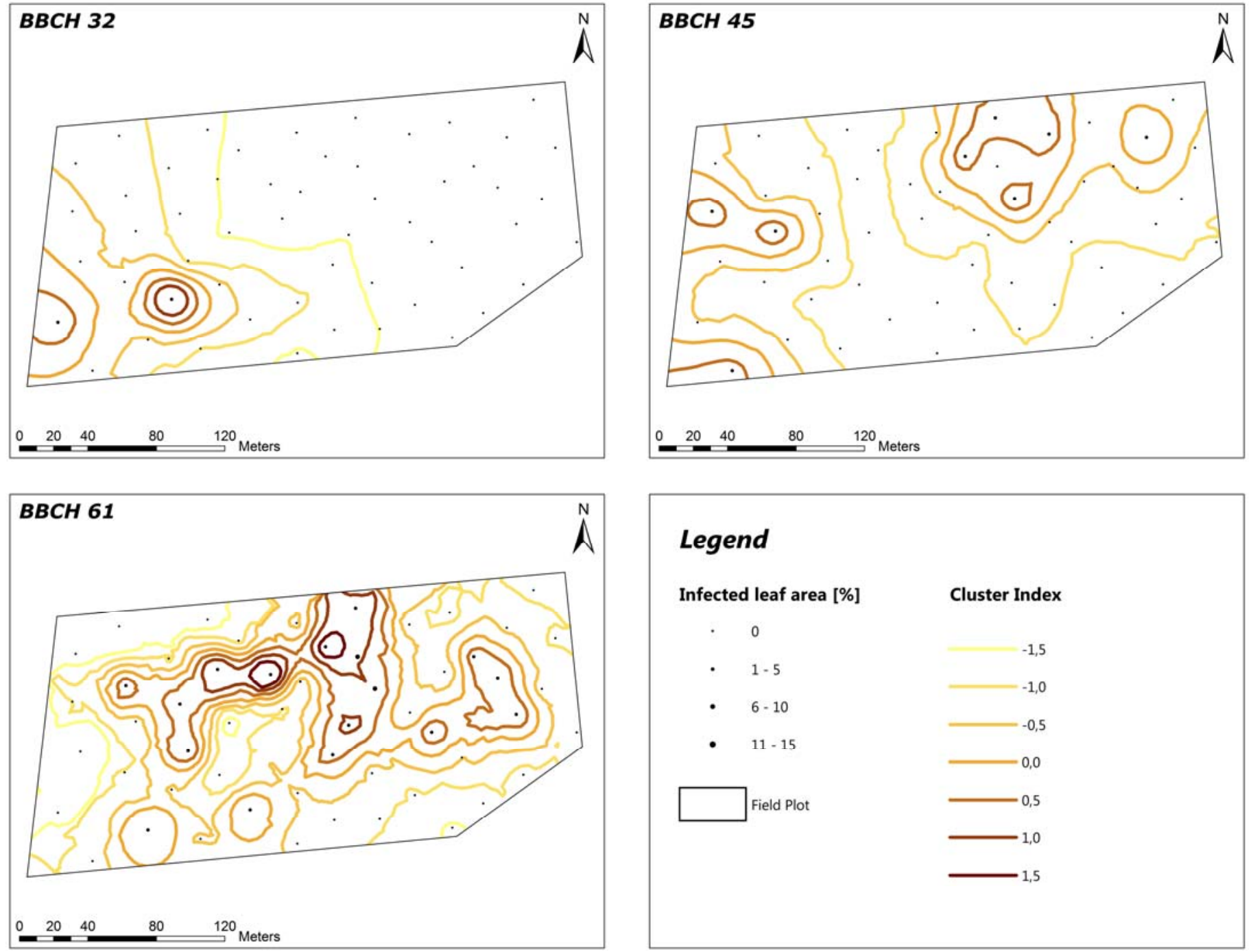

\section{Legend}

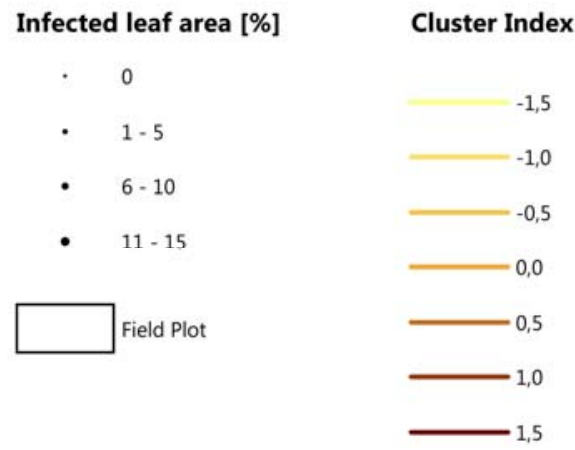

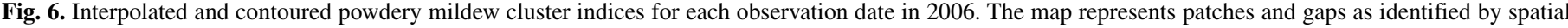

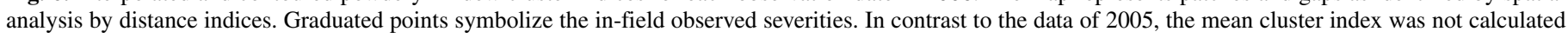
due to a low sampling rate $(n=4)$. 

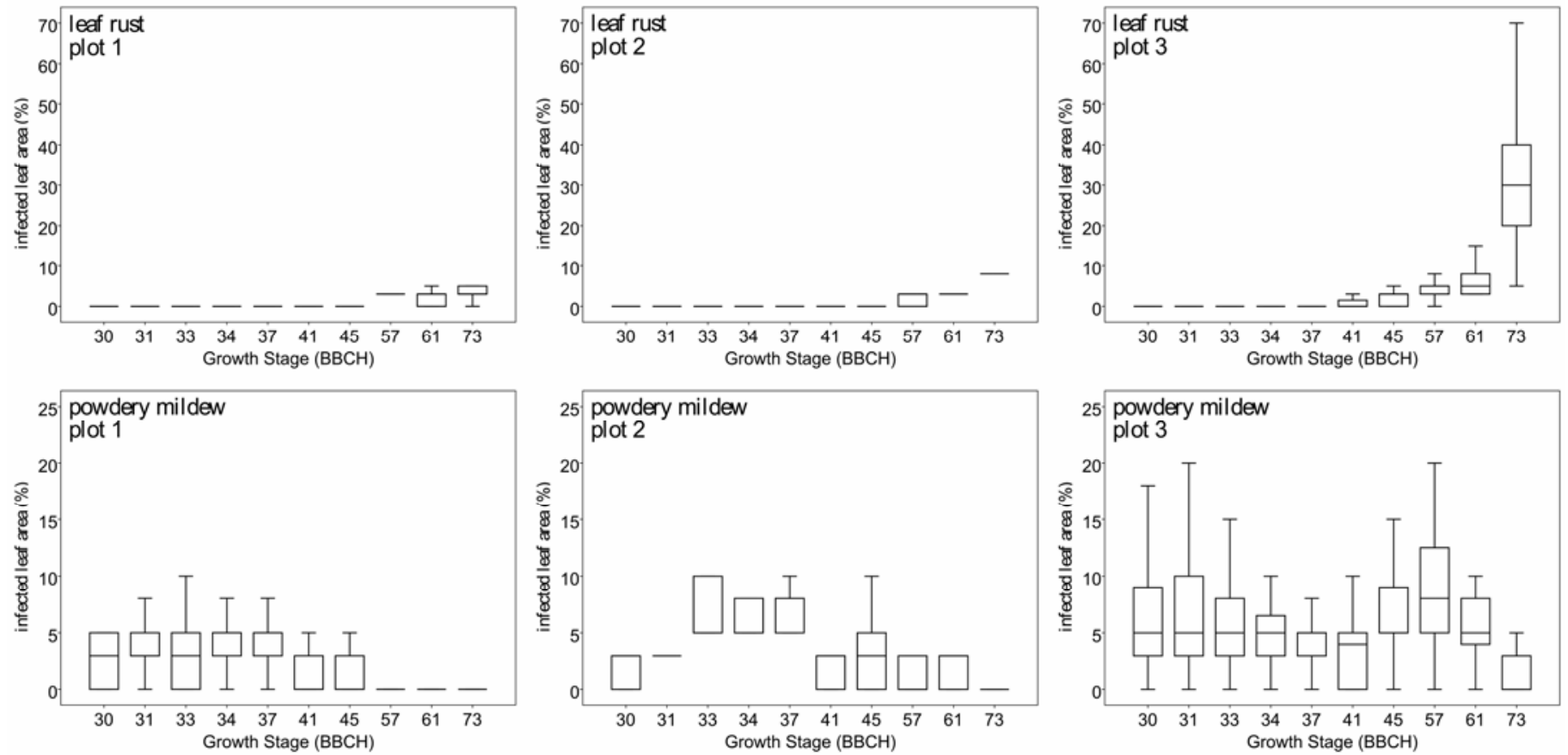

Fig. 7. Boxplots (median, quartiles, and extrema) showing the temporal development of leaf rust and powdery mildew severities in each plot of the observed field in 2005.
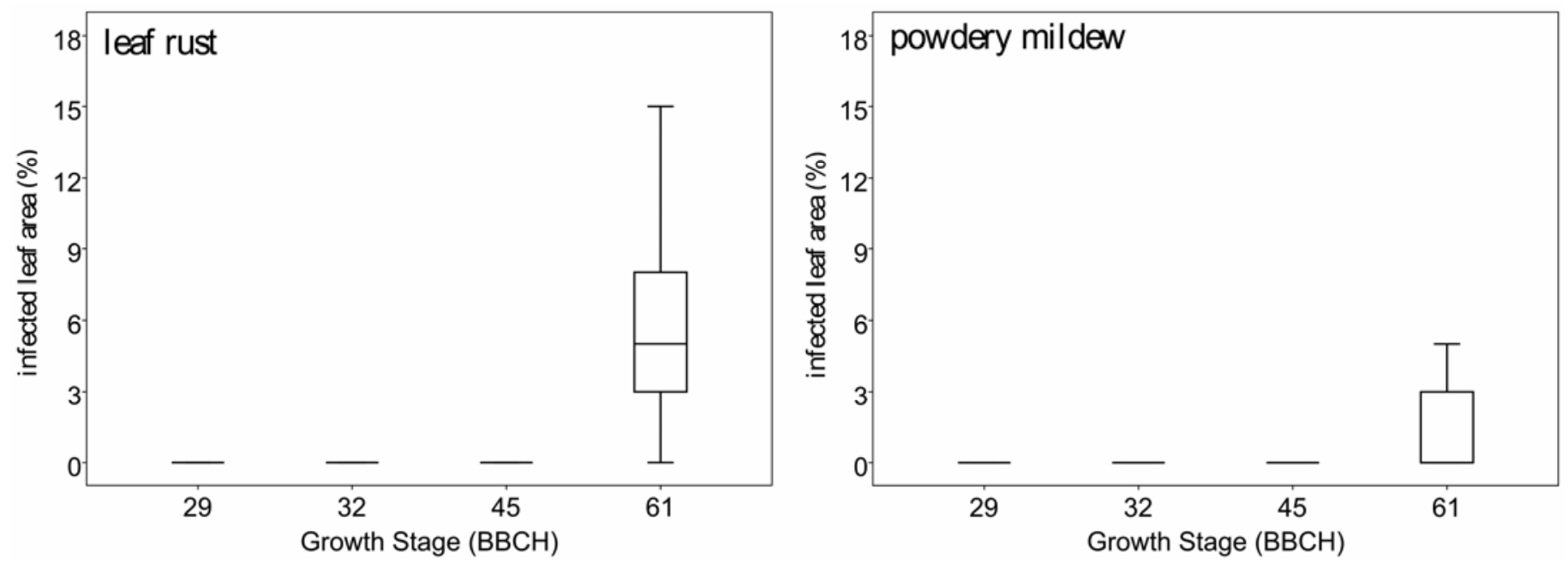

Fig. 8. Boxplots (median, quartiles, and extrema) showing the temporal development of leaf rust and powdery mildew severities of the observed field in 2006.
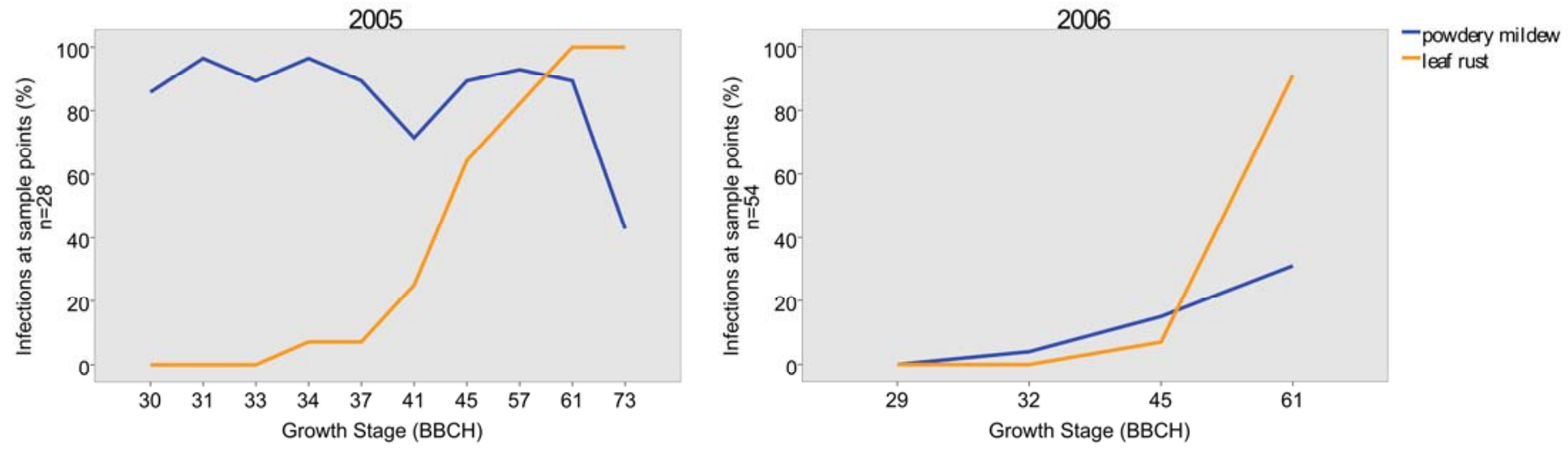

Fig. 9. Incidence (percentages) of leaf rust and powdery mildew observed at sample points in fungicide untreated plot 3 in 2005 and in 2006. 


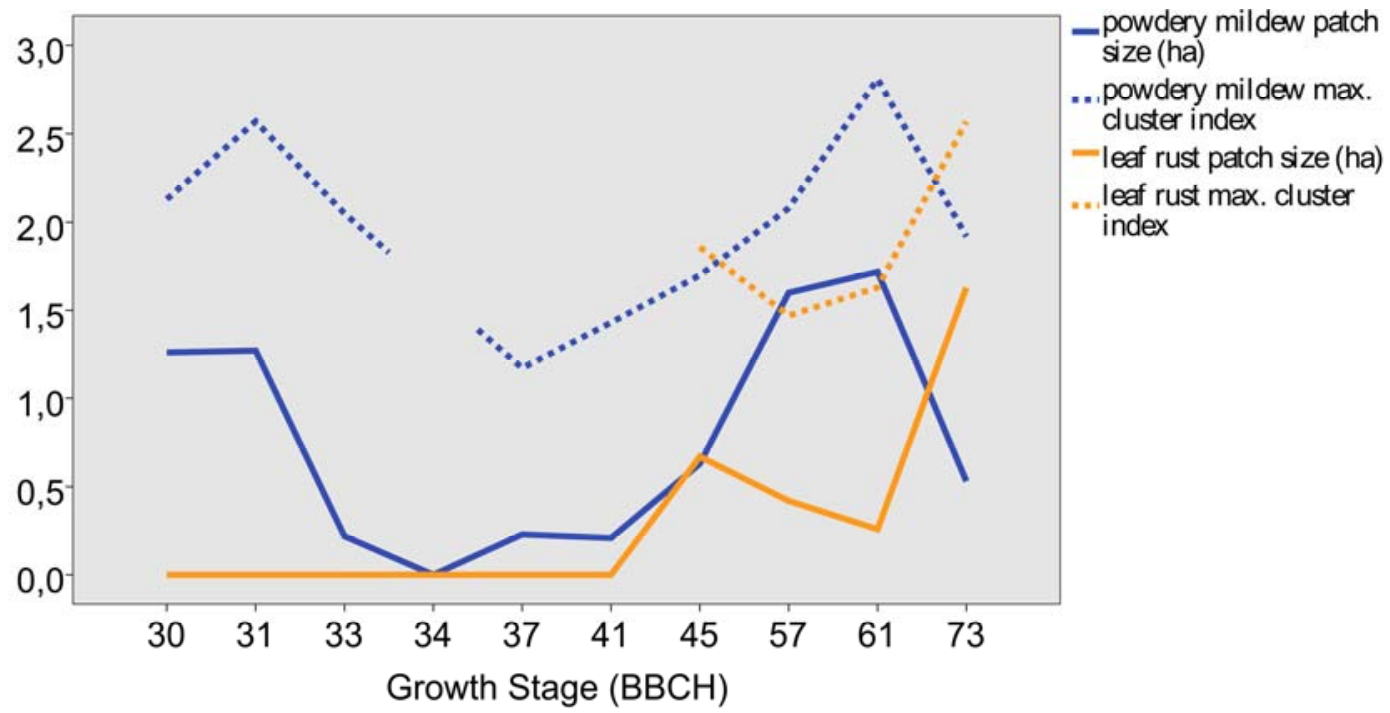

Fig. 10. Course of leaf rust and powdery mildew patch characteristics in 2005.

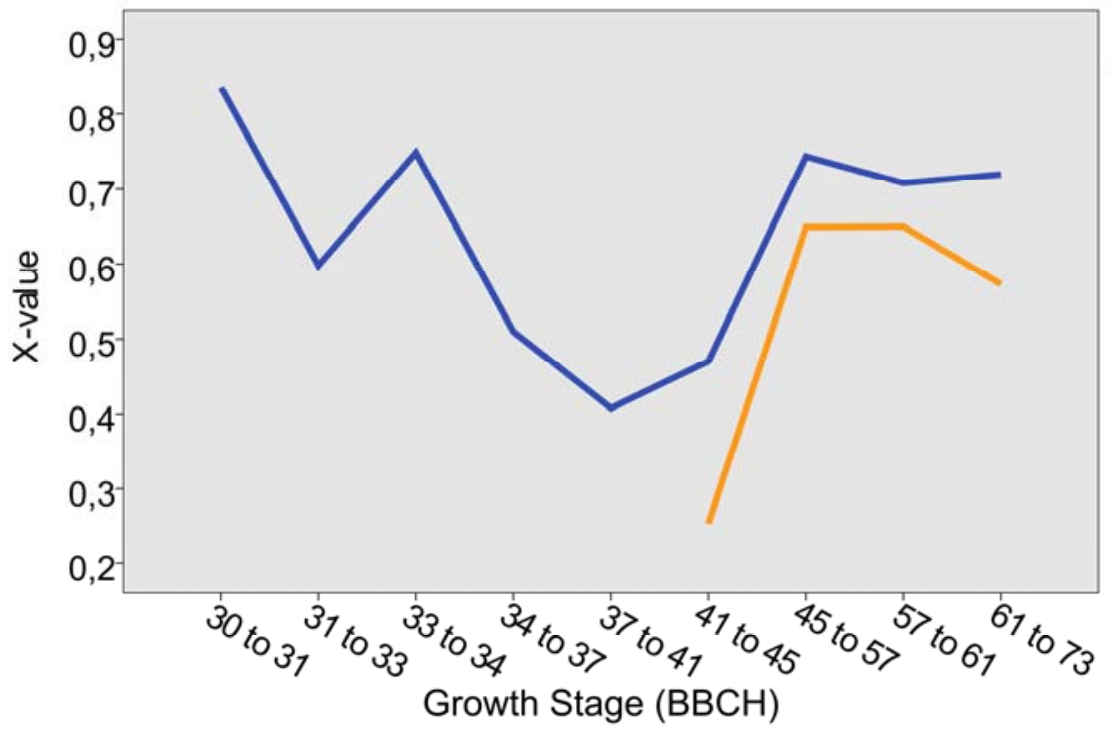

- powdery mildew - leaf rust

Fig. 11. Courses of the overall temporal association of each epidemic (as derived by the spatial analysis by distance indices extension) that demonstrate the temporal correlation of the diseases at certain locations.

inoculum (11). This slowdown of the disease progress can be suitable not only to increase the initial latent period but also to bridge periods with favorable environmental conditions for the pathogens, which would impede disease progress. This particular fact is the most crucial for time-specific control tactics but is not important as long as detailed variations in spatiotemporal dynamics are not considered. Therefore, precision treatment strategies need to be developed on a case-by-case basis. Traditional epidemiological analyses are excellent for describing broad patterns of behavior in epidemics but often miss the detailed variation in dynamics in space and time and, therefore, have to be extended by analyses in high spatial and temporal scales as well as multivariate analyses. For instance, even for leaf rust as a disease with monotonic severity increase, a wide interannual difference occurred that did not allow for a derivation of general optimal disease control dates. Regarding all spatiotemporal facets of both diseases as described, adjusted disease control seems to hold a high potential but requires information on disease-dependent dynamics and prevailing environmental conditions in a high spatial and temporal resolution, which can only be handled by decision support systems.

\section{ACKNOWLEDGMENTS}

This study was carried out under sponsorship of the Research Training Group 722 "Information Techniques for Precision Crop Protection," which is funded by the German Research Foundation (DFG).

\section{LITERATURE CITED}

1. Blackshaw, R. P., and Vernon, R. S. 2006. Spatiotemporal stability of two beetle populations in non-farmed habitats in an agricultural landscape. J. Appl. Ecol. 43:680-689.

2. European Commission. 2002. Agriculture in the European Union, Statistical and Economic Information 2001. Brussels.

3. Everts, K. L., Leath, S., and Finney, P. L. 2001. Impact of powdery mildew and leaf rust on milling and baking quality of soft red winter wheat. Plant Dise. 85:423-429.

4. Ferrandino, F. J. 1989. A distribution-free method for estimating the effect of aggregated plant damage on crop yield. Phytopathology 79:1229-1232.

5. Fitt, B. D. L., Gregory, P. H., Todd, A. D., McCartney, H. A., and MacDonald, O. C. 1987. Spore dispersal and plant disease gradients; a comparison between two empirical models. J. Phytopathol. 118:227-242.

6. Garthwaite, D. G., Thomas, M. R., Anderson, H., and Stoddart, H. 2005. Pesticide usage survey report 202. Arable crops in Great Britain 2004. A 
National Statistic Publication, Department for Environment, Food \& Rural Affairs and Scottish Executive Environment \& Rural Affairs Department. The Food and Environment Research Agency, York, UK.

7. Hunger, B., and Jackson, A. 2004. Foliar Fungicides and Wheat Production in Oklahoma. Oklahoma State University, Cooperative Extension Service. Online publication.

8. Jeger, M. J. 1990. Mathematical analysis and modeling of spatial aspects of plant disease epidemics. Pages 53-95 in: Epidemics of Plant Diseases. Mathematical Analysis and Modeling. J. Kranz, ed. Springer, Berlin, Heidelberg.

9. Kranz, J. 1974. Comparison of epidemics. Annu. Rev. Phytopathol. 12:355-374.

10. Kranz, J. 2003. Comparative Epidemiology of Plant Diseases. Springer, Berlin, Heidelberg, New York, Hong Kong, London, Milan, Paris, Tokyo.

11. Lucas, J. A. 1998. Plant Pathology and Plant Pathogens. Blackwell Publishing, Oxford.

12. Machado, S., Bynum, E. D., Archer, T. L., Bordovsky, J., Rosenow, D. T., Peterson, C., Bronson, K., Nesmith, D. M., Lascano, R. J., Wilson, L. T., and Segarra, E. 2002. Spatial and temporal variability of sorghum grain yield: Influence of soil, water, pests, and diseases relationships. Precis. Agric. 3:389-406.

13. Madden, L. V. 2006. Botanical epidemiology: Some key advances and its continuing role in disease management. Plant disease epidemiology: Facing challenges of the 21st Century. S. Savary and B. M. Cooke, eds. Spec. Issue Eur. J. Plant Pathol. 115:3-23.

14. Madden, L. V., and Campbell, C. L. 1990. Nonlinear disease progress curves. Pages 181-229 in: Epidemics of Plant Diseases. Mathematical Analysis and Modeling. J. Kranz, ed. Springer, Berlin, Heidelberg.

15. Madden, L. V., Hughes, G., and van den Bosch, F. 2007. The Study of Plant Disease Epidemics. The American Phytopathological Society, St. Paul, MN.

16. Moschini, R. C., and Pérez, B. A. 1999. Predicting wheat leaf rust severity using planting date, genetic resistance, and weather variables. Plant Dis. 83:381-384.

17. Mount, M. S., and Slesinski, R. S. 1971. Characteristics of primary development of powdery mildew. Pages 301-322 in: Proc. Int. Symp. Ecol. Leaf Surface Micro-organisms. T. F. Preece and C. H. Dickinson, eds. Academic Press, Newcastle, UK.

18. Nagarajan, S., and Muralidharan, K. 1995. Dynamics of plant diseases. Allied Publisher Limited, New Delhi, Bombay, Calcutta, Madras, Bangalore, Hyderabad, Lucknow, Ahmedabad.

19. Nelson, S. C. 1996. A simple analysis of disease foci. Phytopathology $86: 332-339$

20. Nelson, S. C., and Campbell, C. L. 1993. Comparative spatial analysis of foliar epidemics on winter clover caused by viruses, fungi and a bacterium. Phytopathology 83:288-301.

21. Neudecker, E., Schmidhalter, U., Sperl, C., and Selige, T. 2001. Sitespecific soil mapping by electromagnetic induction. Pages 271-276 in: Proc. 3rd Eur. Conf. Precis. Agric. G. Grenier and S. Blackmore, eds. Agro Montpellier, Montpellier, France.

22. Nutter, F. W., Jr. 1997. Quantifying the temporal dynamics of plant virus epidemics: A review. Crop Prot. 16:603-618.

23. Oerke, E.-C., Dehne, H.-W., Schönbeck, F., and Weber, A. 1994. Crop production and Crop Protection-Estimated Losses in Major Food and Cash Crops. Elsevier Science, Amsterdam, the Netherlands.

24. Parker, S. K., Nutter, F. W., and Gleason, M. L. 1997. Directional spread of Septoria leaf spot in tomato rows. Plant Dis. 81:272-276.

25. Parker, S. R., Shaw, M. W., and Royle, D. J. 1995. The reliability of visual estimated disease severity on cereal leaves. Plant Pathol. 44:856-864.

26. Perry, J. N. 1995. Spatial analysis by distance indices. J. Anim. Ecol. 64:303-314.

27. Perry, J. N. 1998. Measures of spatial pattern for counts. Ecology
79:1008-1017.

28. Perry, J. N., and Dixon, P. M. 2002. A new method to measure spatial association for ecological count data. Ecoscience 9:133-141.

29. Perry, J. N., Winder, L., Holland, J. M., and Alston, R. D. 1999. Red-blue plots for detection of cluster in count data. Ecol. Lett. 2:106-113.

30. Pethybridge, S. J., Esker, P., Hay, F., Wilson, C., and Nutter, F. W. 2005. Spatiotemporal description of epidemics caused by Phoma ligulicola in Tasmanian Pyrethrum fields. Phytopathology 95:648-658.

31. Pethybridge, S. J., and Turechek, W. W. 2003. Analysis of the association among three viruses infecting hop in Australia. Plant Pathol. 52:158167.

32. Quinlan, J. R. 1993. C4.5: Programs for Machine Learning. Morgan Kaufmann, San Mateo, CA

33. Roelfs, A. P. 1972. Gradients in horizontal dispersal of cereal rust uredospores. Phytopathology 62:70-76.

34. Savary, S., and Cooke, B. M. 2006. Foreword. Plant disease epidemiology: Facing challenges of the 21st Century. S. Savary and B. M. Cooke, eds. Spec. Issue Eur. J. Plant Pathol. 115:1-2.

35. Scott, K. J., and Chakravorty, A. K., eds. 1982. The Rust Fungi. Academic Press, London, New York, Paris, San Diego, San Francisco, Sao Paulo, Sydney, Tokyo, Toronto

36. Shah, D. A., Bergstrom, G. C., and Ueng, P. P. 2001. Foci of Stagonospora nodorum blotch in winter wheat before canopy development. Phytopathology 91:642-647.

37. Thomas, C. F. G., Parkinson, L., Griffiths, G. J. K., Fernandez Garcia, A., and Marshall, E. J. P. 2001. Aggregation and temporal stability of carabid beetle distributions in field and hedgerow habitats. J. Appl. Biol. 38:100116.

38. Tubajika, K. M., Civerolo, E. L., Ciomperlik, M. A., Luvisi, D. A., and Hashim, J. M. 2004. Analysis of the spatial patterns of Pierce's disease incidence in the lower San Joaquin Valley in California. Phytopathology 94:1136-1144.

39. Van Drooge, H. L., Groeneveld, C. N., and Schipper, H. J. 2001. Data on application frequency of pesticide for risk assessment purposes. Ann. Occup. Hyg. 45(1001):95-101.

40. Warner, D. J., Allen-Williams, L. J., Warrington, W., Ferguson, A. W., and Williams, I. H. 2003. Mapping, characterisation, and comparison of the spatiotemporal distributions of cabbage stem flea beetle (Psylliodes chrysocephala), carabids, and Collembola in a crop of winter oilseed rape (Brassica napus). Entomol. Exp. Appl. 109:225-234.

41. West, J. S., Bravo, C., Oberti, R., Lemaire, D., Moshou, D., and McCartney, H. A. 2003. The potential of optical canopy measurement for targeted control of field crop diseases. Annu. Rev. Phytopathol. 41:593614.

42. West, J. S., and McCartney, H. A. 2002. Optical disease detection and estimation of latent infections around disease foci for targeted pesticide application. Asp. Appl. Biol. 66:463-468.

43. Winder, L., Alexander, C. J., Holland, J. M., Woolley, C., and Perry, J. N. 2001. Modelling the dynamic spatiotemporal response of predators to transient prey patches in the field. Ecol. Lett. 4(6):568-576.

44. Xu, X. M., and Madden, L. V. 2004. Use of SADIE statistics to study spatial dynamics of plant disease epidemics. Plant Pathol. 53:38-49.

45. Xu, X. M., and Ridout, M. S. 1998. Effects of initial epidemic conditions, sporulation rate, and spore dispersal gradient on the spatiotemporal dynamics of plant disease epidemics. Phytopathology 88:10001012.

46. Zadoks, J. C., Chang, T. T., and Konzak, C. F. 1974. A decimal code for growth stage of cereal. Weed Res. 14:415-421.

47. Zadoks, J. C., and Schein, R. D. 1979. Epidemiology and Plant Disease Management. Oxford University Press, New York, Oxford.

48. Zadoks, J. C., and van den Bosch, F. 1994. On the spread of plant disease: A theory on foci. Annu. Rev. Phytopathol. 32:503-521. 